\title{
Hunting for QCD strings in $e^{+} e^{-}$-annihilation
}

\author{
Marina Kozhevnikova ${ }^{1, *}$, Armen Oganesian ${ }^{2, * *}$, and Oleg Teryaev ${ }^{1, * * *}$ \\ ${ }^{1}$ Joint Institute for Nuclear Research, Dubna, Russia \\ ${ }^{2}$ Institute of Theoretical and Experimental Physics, Moscow, Russia
}

\begin{abstract}
We develop a new mathematical method of extraction of nonperturbative corrections in QCD. The main attention is directed to the study of dimension 2 operator and its properties. The ordinary perturbation theory (PT) and analytical perturbation theory (APT) are used. The $D$-function is explored in dependence on both $Q^{2}$ and Borel parameter $M^{2}$. It is shown that the $C_{2}$ coefficient of the dimension 2 operator is negative and its compatibility to zero depends on choice of PT or APT and can vary in chosen ranges of $M^{2}$. The strong (anti)correlation between $C_{2}$ and gluon condensate found previously is confirmed.
\end{abstract}

\section{Introduction}

The problem of the existence of an operator with dimension 2 whose contribution to the QCD sum rules [1] is proportional to $1 / Q^{2}$ and the search for relevant corrections of the operator product expansion (OPE) have already been studied for quite a long time [2-4]. In the pioneering paper [2] the concept of short strings leading to corrections with dimension 2 was suggested. In the Cornell potential [5] $V(r) \approx-\frac{4 \alpha_{s}(r)}{3 r}+k r$ the term $k r$ describes the string potential (connected to the phenomenon of confinement) at short distances leading to the correction $\sim k / Q^{2}$. Note that in local OPE the first correction to the $e^{+} e^{-}$-annihilation cross-section is $\sim\left\langle G_{\mu \nu} G^{\mu \nu}\right\rangle / Q^{4}$. The contribution of the operator with dimension 2 to the $e^{+} e^{-}$data was studied later $[4,6]$ and it was found to be compatible to zero with large errors. The main purpose is to verify whether the operator with dimension 2 exists.

We extend our analysis [7] based on the use of the Adler function, applying the Borel transform by including the consideration of APT [8-15].

\section{Construction of the model of the data}

We get the separate fits of the data on the following channels: $e^{+} e^{-} \rightarrow \pi^{+} \pi^{-}[16] ; e^{+} e^{-} \rightarrow 2 \pi^{+} 2 \pi^{-}$[17]; $e^{+} e^{-} \rightarrow \pi^{+} \pi^{-} 2 \pi^{0}$ [18-23]; $e^{+} e^{-} \rightarrow 3 \pi^{+} 3 \pi^{-}$[24]; $e^{+} e^{-} \rightarrow 2 \pi^{+} 2 \pi^{-} 2 \pi^{0}[24]$.

For fitting of each process we use the method of minimization of $\chi^{2}$-functional. The data for the process $e^{+} e^{-} \rightarrow \pi^{+} \pi^{-}$is presented as squared form factor on energy $\sqrt{s}$. To fit the data we use [7] the generalized [16] model of Gounaris and Sakurai [25].

\footnotetext{
*e-mail: kozhevnikova@jinr.ru

**e-mail: armen@itep.ru

***e-mail: teryaev@theor.jinr.ru
} 
The fitting results are shown in Tab. 1. We use the PDG [26] data for $m_{\rho}, m_{\omega}$ and $\Gamma_{\omega}$. In calculation of $\chi^{2}$ only statistical errors are used.

Table 1. The fitting results for the process $e^{+} e^{-} \rightarrow \pi^{+} \pi^{-}$

\begin{tabular}{c|ccccc} 
& $V$ & $M_{V}, \mathrm{GeV}$ & $\Gamma_{V}, \mathrm{GeV}$ & $\alpha_{V}$ & $d$ \\
\hline$e^{+} e^{-} \rightarrow \pi^{+} \pi^{-}$ & $\rho$ & $\mathbf{0 . 7 7 5}$ (PDG) & $0.148 \pm 0.002$ & 1 & \\
$\chi_{\min }^{2}=1.05$ & $\omega$ & $\mathbf{0 . 7 8 2}$ (PDG) & $\mathbf{0 . 0 0 8}$ (PDG) & $0.0020 \pm 0.0003$ & $0.408 \pm 0.041$ \\
& $\rho^{\prime}$ & $1.353 \pm 0.024$ & $0.328 \pm 0.149$ & $-0.085 \mp 0.005$ &
\end{tabular}

For the cross-sections of the other processes we introduce the model including two or three Gaussian resonances [7]. The fitting results are shown in Tab. 2.

Table 2. The fitting results for particular $e^{+} e^{-}$-annihilation channels

\begin{tabular}{c|cccc} 
& $i$ & $M_{i}, \mathrm{GeV}$ & $\sigma_{i}, \mathrm{GeV}$ & $\alpha_{i}$ \\
\hline$e^{+} e^{-} \rightarrow 2 \pi^{+} 2 \pi^{-}$ & 1 & $1.512 \pm 0.003$ & $0.242 \pm 0.002$ & $1.560 \pm 0.016$ \\
$\chi_{\min }^{2}=1.85$ & 2 & $2.125 \pm 0.016$ & $0.231 \pm 0.012$ & $0.458 \pm 0.022$ \\
& 3 & $2.656 \pm 0.025$ & $0.808 \pm 0.019$ & $0.590 \pm 0.014$ \\
\hline$e^{+} e^{-} \rightarrow \pi^{+} \pi^{-} 2 \pi^{0}$ & $i$ & $M_{i}, \mathrm{GeV}$ & $\sigma_{i}, \mathrm{GeV}$ & $\alpha_{i}$ \\
$\chi_{\min }^{2}=8.45$ & 2 & $1.786 \pm 0.005$ & $0.327 \pm 0.003$ & $1.484 \pm 0.029$ \\
& 3 & $1.343 \pm 0.004$ & $0.188 \pm 0.004$ & $0.916 \pm 0.012$ \\
\hline$e^{+} e^{-} \rightarrow 3 \pi^{+} 3 \pi^{-}$ & $i$ & $M_{i}, \mathrm{GeV}$ & $\sigma_{i}, \mathrm{GeV}$ & $\alpha_{i}$ \\
\hline$\chi_{\min }^{2}=0.62$ & 1 & $1.789 \pm 0.015$ & $0.083 \pm 0.012$ & $0.154 \pm 0.020$ \\
& 2 & $2.050 \pm 0.014$ & $0.291 \pm 0.011$ & $0.433 \pm 0.017$ \\
\hline$e^{+} e^{-} \rightarrow 2 \pi^{+} 2 \pi^{-} 2 \pi^{0}$ & $i$ & $M_{i}, \mathrm{GeV}$ & $\sigma_{i}, \mathrm{GeV}$ & $\alpha_{i}$ \\
\hline$\chi_{\min }^{2}=1.03$ & 1 & $2.348 \pm 0.012$ & $0.331 \pm 0.008$ & $1.598 \pm 0.085$ \\
& 2 & $1.740 \pm 0.011$ & $0.120 \pm 0.009$ & $0.558 \pm 0.035$
\end{tabular}

\section{Calculation of $R$-ratio and $D$-function}

Collecting all the data, we calculate the $I=1 R$-ratio whose theoretical form in the PT and in the APT is:

$$
R_{\mathrm{th}}^{\mathrm{PT} / \mathrm{APT}}(s)=\frac{3}{2}\left(1+\frac{\alpha_{s}^{\mathrm{PT} / \mathrm{APT}}(s)}{\pi}\right) .
$$

The summation result is presented in Fig. 1 by the black solid curve. Blue dots correspond to particular annihilation channels. The $R$-ratio in the full energy region is $R_{\text {exp-th }}(s)=R_{\text {exp }}(s) \theta\left(s \leq s_{0}\right)+R_{\text {th }}(s) \theta\left(s>s_{0}\right)$, where $s_{0}$ is the continuum threshold, we calculated the value $s_{0} \approx 1.54^{2} \mathrm{GeV}^{2}$.

To calculate the error of the full $R$-ratio, we find the error of the full cross-section and then in the interval of $s$ from 0 to $s_{0}$ we find: $\Delta R(s)=\Delta \sigma_{e^{+} e^{-} \rightarrow \text { pions }}(s) / \sigma_{e^{+} e^{-} \rightarrow \mu^{+} \mu^{-}}(s)$. To the full ${ }^{1}$ error of $\sigma$ we account for systematic ones by adding and subtracting them at each point $s_{i}$ to get the minimal and maximal value of $\sigma\left(s_{i}\right)$. Then we fit each of the three obtained sets (average, minimal and maximum values) and get three fitting curves. In the region of $s$ from $s_{0}$ to $\infty$ where the $R$-ratio is described by the theoretical form (1) its error is neglected.

\footnotetext{
${ }^{1}$ The contribution of the statistical errors is very small since it is proportional to $1 / N_{\text {d.f. }}$, so we neglect it.
} 


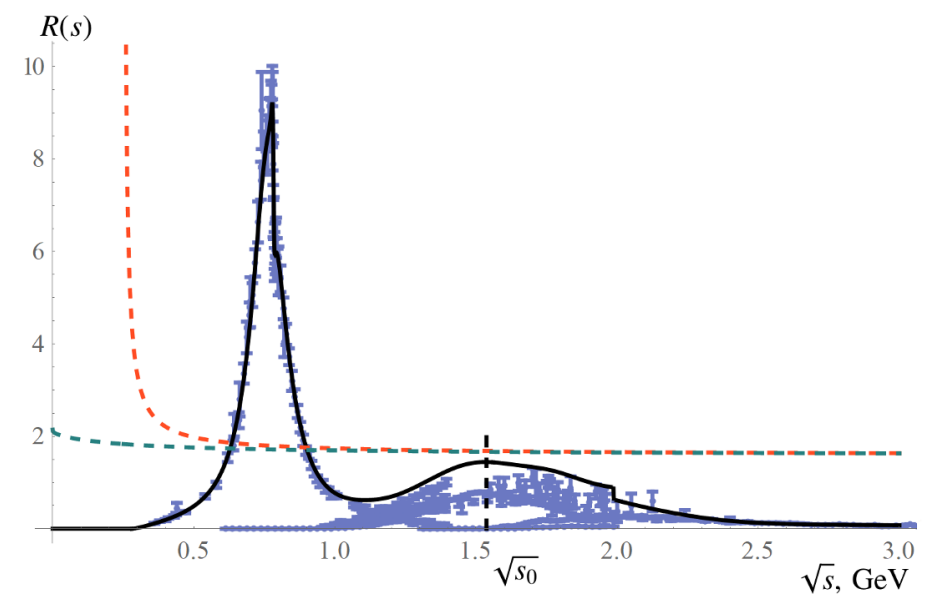

Figure 1. The full $R$-ratio $\left(R_{\exp }\right)$ in dependence on $\sqrt{s}$ at $\sqrt{s} \leq 3 \mathrm{GeV}$ (black), the experimental data (blue dots), the theoretical representation $R_{\mathrm{th}}(s)$ in the PT (red, dashed) and in the APT (blue, dashed). $s_{0} \approx 1.54^{2} \mathrm{GeV}^{2}$

There exist two ways to write down $D$-function, dispersional: $D_{\exp }\left(Q^{2}\right)=$ $Q^{2} \int_{4 m_{\pi}^{2}}^{\infty} \frac{R_{\text {exp-th }}(s) d s}{\left(s+Q^{2}\right)^{2}}$, and by PT and OPE: $D_{\mathrm{PT}+\mathrm{OPE}}\left(Q^{2}\right)=\frac{3}{2}\left[1+\frac{\alpha_{s}\left(Q^{2}\right)}{\pi}+\sum_{n \geq 1} \Gamma(n) \frac{C_{2 n}}{Q^{2 n}}\right]$, and similarly for APT with $\alpha_{s}\left(Q^{2}\right) \rightarrow \mathcal{A}_{s}\left(Q^{2}\right)$ and $C_{2 n} \rightarrow \tilde{C}_{2 n}$, in the present work we use three first corrections. The factor $\Gamma(n)$ is introduced in order to have the simple expression for Borel transform of $D$-function.

The running coupling in one-loop approximation has the form: $\alpha_{s}\left(Q^{2}\right)=\frac{4 \pi}{b_{0}} \frac{1}{\ln \left(Q^{2} / \Lambda^{2}\right)}$. Here $b_{0}=11-2 N_{f} / 3$ is the first coefficient of the $\beta$-function expansion and $\Lambda$ is the QCD scale parameter. Note that $\alpha_{s}\left(Q^{2}\right)$ has the unphysical singularity at $Q^{2}=\Lambda^{2}$. In the framework of APT the running coupling reads: $\mathcal{A}_{s}\left(Q^{2}\right)=\frac{4 \pi}{b_{0}}\left[\frac{1}{\ln \left(Q^{2} / \Lambda_{\mathrm{APT}}^{2}\right)}-\frac{\Lambda_{\mathrm{APT}}^{2}}{Q^{2}-\Lambda_{\mathrm{APT}}^{2}}\right]$. One can see that the additional term in this expression removes the singularity.

\section{The Borel transform of $D$-function and sum rules}

The Borel transformed forms of the $D$-function ${ }^{2}$, dispersional and by OPE, are:

$$
\begin{aligned}
& \hat{B}_{Q^{2} \rightarrow M^{2}}\left[D_{\exp }\left(Q^{2}\right)\right]=\Phi_{\exp }\left(M^{2}\right)=\int_{0}^{\infty} R_{\text {exp-th }}(s)\left(1-\frac{s}{M^{2}}\right) e^{-s / M^{2}} \frac{d s}{M^{2}}, \\
& \hat{B}_{Q^{2} \rightarrow M^{2}}\left[D_{\mathrm{PT}+\mathrm{OPE}}\left(Q^{2}\right)\right]=\Phi_{\mathrm{PT}+\mathrm{OPE}}\left(M^{2}\right)=\frac{3}{2} \frac{\hat{B}_{Q^{2} \rightarrow M^{2}}\left[\alpha_{s}\left(Q^{2}\right)\right]}{\pi}+\frac{3}{2}\left(\frac{C_{2}}{M^{2}}+\frac{C_{4}}{M^{4}}+\frac{C_{6}}{M^{6}}\right),
\end{aligned}
$$

and similarly for APT with $\alpha_{s}\left(Q^{2}\right) \rightarrow \mathcal{A}_{s}\left(Q^{2}\right)$ and $C_{2 n} \rightarrow \widetilde{C}_{2 n}$.

Equating the two forms of the Adler function, we get the sum rules for PT and APT.

The error of $\Phi\left(M^{2}\right), \Delta \Phi\left(M^{2}\right)$, can be calculated using formula (2) through the error of the $R$-ratio, $\Delta R(s): \Delta \Phi\left(M^{2}\right)=\int_{0}^{s_{0}} \Delta R(s)\left(1-\frac{s}{M^{2}}\right) e^{-s / M^{2}} \frac{d s}{M^{2}}$.

\footnotetext{
${ }^{2}$ Note that the leading order of perturbative QCD terms does not contribute.
} 


\section{Analysis of the sum rules and extraction of the condensates}

The coefficients $C_{2}, C_{4}{ }^{3}$ are extracted from obtained sum rules using the $\chi^{2}$-minimization:

$$
\chi^{2}\left(C_{2}, C_{4}\right)=\frac{1}{N_{\text {d.f. }}} \sum_{n=1}^{N} \frac{\left(\Phi_{\exp }\left(M_{n}^{2}\right)-\Phi_{\mathrm{PT}(\mathrm{APT})+\mathrm{OPE}}\left(M_{n}^{2} ; C_{2}, C_{4}\right)\right)^{2}}{\Delta \Phi_{\exp }\left(M_{n}^{2}\right)^{2}},
$$

where $N_{\text {d.f. }}=N-d$ is the number of degrees of freedom, $N$ is the number of points, $d=2$ is the number of varying parameters. To explore the dependence on the squared Borel mass $M^{2}$, we consider the different ranges of $M^{2}$ obtained by dividing the full range by equidistant points. We perform the $\chi^{2}$-minimization on each range. We implement this method for PT and APT.

The results for PT and APT with $\Lambda=0.25 \mathrm{GeV}$ are shown in Tabs. 3 and 4 consequently and in Fig. 2. The main qualitative feature of all received results is the strong (anti)correlation between $C_{2}$ and gluon condensate (g.c.), expressed as g.c. $\left(\mathrm{GeV}^{4}\right)=A\left(\mathrm{GeV}^{2}\right) \cdot C_{2}\left(\mathrm{GeV}^{2}\right)+B\left(\mathrm{GeV}^{4}\right)$, marked previously in our work [7]. One can see that at one $\sigma$-level our results for standard local gluon condensate are compatible with that from $[1,27,28]$.

Table 3. The fitting results for different intervals of $M^{2}$ in PT with $\Lambda=0.25 \mathrm{GeV}$. In the fifth column the $\sigma$-level where $C_{2}=0$ is shown. In the sixth column the (anti)correlation between the gluon condensate and $C_{2}$ is shown

\begin{tabular}{cccccc} 
Range of $M^{2}, \mathrm{GeV}^{2}$ & $C_{2}, \mathrm{GeV}^{2}$ & $\frac{<\alpha_{s} G G>}{\pi}, \mathrm{GeV}^{4}$ & $\chi^{2}$ & $\sigma$-level & (Anti)correlation \\
\hline$[10 / 20,160 / 20]$ & $-0.093 \mp 0.054$ & $0.025 \pm 0.008$ & 0.758 & 3 & $-0.153 C_{2}+0.011$ \\
\hline$[11 / 20,120 / 20]$ & $-0.076 \mp 0.052$ & $0.023 \pm 0.008$ & 0.553 & 3 & $-0.154 C_{2}+0.011$ \\
\hline$[12 / 20,100 / 20]$ & $-0.065 \mp 0.052$ & $0.021 \pm 0.008$ & 0.406 & 2 & $-0.154 C_{2}+0.011$ \\
\hline$[13 / 20,90 / 20]$ & $-0.058 \mp 0.053$ & $0.020 \pm 0.008$ & 0.323 & 2 & $-0.154 C_{2}+0.011$ \\
\hline$[14 / 20,80 / 20]$ & $-0.052 \mp 0.053$ & $0.019 \pm 0.008$ & 0.265 & 1 & $-0.155 C_{2}+0.011$ \\
\hline$[15 / 20,70 / 20]$ & $-0.047 \mp 0.052$ & $0.018 \pm 0.008$ & 0.212 & 1 & $-0.155 C_{2}+0.011$ \\
\hline$[16 / 20,60 / 20]$ & $-0.042 \mp 0.051$ & $0.017 \pm 0.008$ & 0.156 & 1 & $-0.155 C_{2}+0.011$ \\
\hline$[17 / 20,50 / 20]$ & $-0.037 \mp 0.048$ & $0.016 \pm 0.007$ & 0.097 & 1 & $-0.156 C_{2}+0.011$ \\
\hline$[18 / 20,40 / 20]$ & $-0.032 \mp 0.044$ & $0.016 \pm 0.007$ & 0.041 & 1 & $-0.156 C_{2}+0.011$ \\
\hline$[19 / 20,30 / 20]$ & $-0.027 \mp 0.036$ & $0.015 \pm 0.006$ & 0.005 & 1 & $-0.156 C_{2}+0.011$
\end{tabular}

To compare the method of Borel transform with the standard analysis of $D$-function, we get the $D$-function in dependence of $Q^{2}$ with the parameters $C_{2}$ and $C_{4}$ obtained using (3) in range $0.5 \mathrm{GeV}^{2} \leq M^{2} \leq 8 \mathrm{GeV}^{2}$. The results are shown in Fig. 3. The OPE curve is in reasonable agreement with dispersion curve at $Q \geq 1 \mathrm{GeV}$.

\section{Results}

The model of the data on $e^{+} e^{-}$-annihilation is constructed, the $R$-ratio and $D$-function are calculated, the Borel transform is applied, the condensates are extracted.

The PT and APT approaches are compared and it is shown that the contribution of short string is closer to zero for APT.

The observed earlier (anti)correlation between short string and gluon condensate is confirmed.

\footnotetext{
${ }^{3} \mathrm{We}$ fix the coefficient $C_{6}$ calculating quark condensate [1] as in work [27]: $C_{6}=-\frac{448 \pi^{3}}{27} \alpha_{s}\langle\bar{q} q\rangle^{2} \approx$ $-0.116 \mathrm{GeV}^{6}$.
} 
Table 4. The fitting results for different intervals of $M^{2}$ in APT with $\Lambda=0.25 \mathrm{GeV}$. In the fifth column the $\sigma$-level where $\widetilde{C}_{2}=0$ is shown. In the sixth column the (anti)correlation between the gluon condensate and $\widetilde{C}_{2}$ is shown

\begin{tabular}{cccccc} 
Range of $M^{2}, \mathrm{GeV}^{2}$ & $\widetilde{C}_{2}, \mathrm{GeV}^{2}$ & $\frac{<\alpha_{s} G G>}{\pi}, \mathrm{GeV}^{4}$ & $\chi^{2}$ & $\sigma$-level & (Anti)correlation \\
\hline$[10 / 20,160 / 20]$ & $-0.067 \mp 0.053$ & $0.026 \pm 0.008$ & 0.723 & 2 & $-0.159 \widetilde{C}_{2}+0.016$ \\
\hline$[11 / 20,120 / 20]$ & $-0.048 \mp 0.053$ & $0.023 \pm 0.008$ & 0.508 & 1 & $-0.159 \widetilde{C}_{2}+0.016$ \\
\hline$[12 / 20,100 / 20]$ & $-0.036 \mp 0.054$ & $0.021 \pm 0.009$ & 0.368 & 1 & $-0.159 \widetilde{C}_{2}+0.016$ \\
\hline$[13 / 20,90 / 20]$ & $-0.028 \mp 0.057$ & $0.020 \pm 0.009$ & 0.296 & 1 & $-0.159 \widetilde{C}_{2}+0.016$ \\
\hline$[14 / 20,80 / 20]$ & $-0.022 \mp 0.058$ & $0.019 \pm 0.009$ & 0.244 & 1 & $-0.159 \widetilde{C}_{2}+0.016$ \\
\hline$[15 / 20,70 / 20]$ & $-0.017 \mp 0.059$ & $0.018 \pm 0.009$ & 0.195 & 1 & $-0.159 \widetilde{C}_{2}+0.016$ \\
\hline$[16 / 20,60 / 20]$ & $-0.012 \mp 0.058$ & $0.017 \pm 0.009$ & 0.142 & 1 & $-0.159 \widetilde{C}_{2}+0.016$ \\
\hline$[17 / 20,50 / 20]$ & $-0.006 \mp 0.055$ & $0.017 \pm 0.009$ & 0.086 & 1 & $-0.160 \widetilde{C}_{2}+0.016$ \\
\hline$[18 / 20,40 / 20]$ & $-0.000 \mp 0.051$ & $0.016 \pm 0.008$ & 0.035 & 1 & $-0.160 \widetilde{C}_{2}+0.016$ \\
\hline$[19 / 20,30 / 20]$ & $0.006 \mp 0.045$ & $0.015 \pm 0.007$ & 0.004 & 1 & $-0.160 \widetilde{C}_{2}+0.016$
\end{tabular}

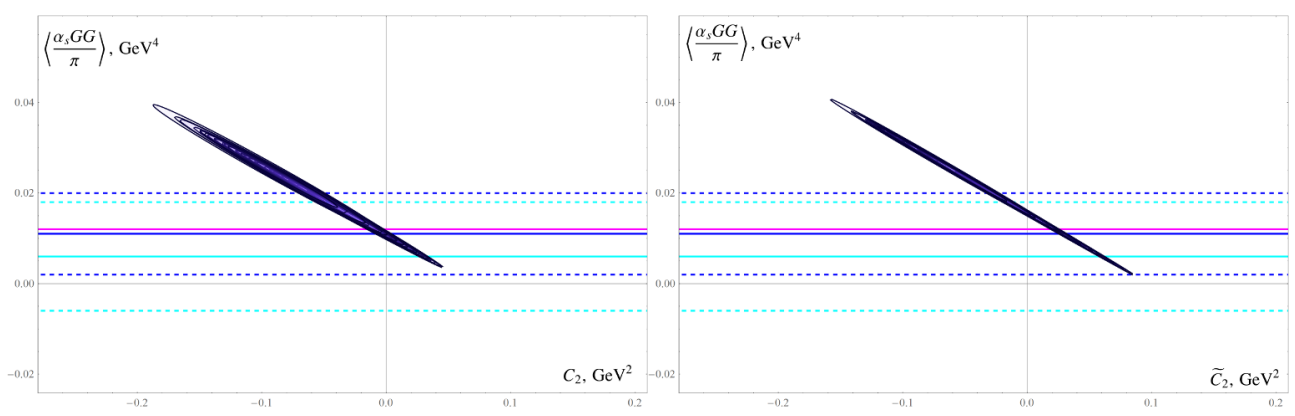

Figure 2. The regions of $\chi^{2} \leq \chi_{\min }^{2}+1, \chi^{2} \leq \chi_{\min }^{2}+2, \chi^{2} \leq \chi_{\min }^{2}+3$ for PT (left panel) and APT (right panel) with the dependence of the gluon condensate on $C_{2}$ with $\Lambda=0.25 \mathrm{GeV}$

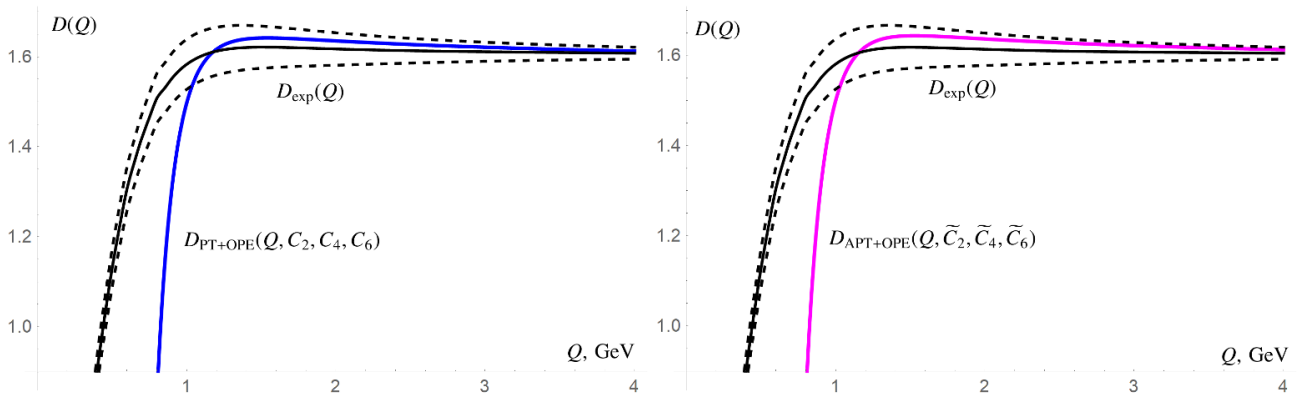

Figure 3. The standard dependence of $D$-function on $Q$ for PT (left panel) with received values of $C_{2}$ and $C_{4}$ (blue) and for APT (right panel) with $\widetilde{C}_{2}$ and $\widetilde{C}_{4}$ (magenta) and dispersion curves (black) with taking into account dominant systematic error (statistic error is negligible)

The standard dependence $D\left(Q^{2}\right)$ with the received parameters $C_{2}$ and $C_{4}$ is in a good agreement with the dispersional form at $Q \geq 1 \mathrm{GeV}$. 
Acknowledgments. We are indebted to O.P. Solovtsova, D.V. Dedovich for useful discussions. The work of O.T. was supported by RFBR grant 18-02 01107.

\section{References}

[1] M. A. Shifman, A. I. Vainshtein and V.I. Zakharov, Nucl. Phys. B 147, 385 and 448 (1979)

[2] K.G. Chetyrkin, S. Narison, V.I. Zakharov. Nucl. Phys. B 550, 353 (1999)

[3] S. Narison, V.I. Zakharov. Phys. Lett. B 679, 355 (2009)

[4] S. Bodenstein, C.A. Dominguez, S.I. Eidelman, H. Spiesberger, K. Schilcher. JHEP 1201, 039 (2012)

[5] E. Eichten, K. Gottfried, T. Kinoshita, J. B. Kogut, K. D. Lane and T. M. Yan, Phys. Rev. Lett. 34, 369 (1975). Erratum: [Phys. Rev. Lett. 36, 1276 (1976)]

[6] H. Spiesberger, Mod. Phys. Lett. A 28, 1360009 (2013)

[7] M. E. Kozhevnikova, A. G. Oganesian and O. V. Teryaev, EPJ Web Conf. 138, 02006 (2017)

[8] V. L. Khandramai, R. S. Pasechnik, D. V. Shirkov, O. P. Solovtsova and O. V. Teryaev, Phys. Lett. B 706, 340 (2012)

[9] O. P. Solovtsova, Nonlin. Phenom. Complex Syst. 17, 445 (2014)

[10] D. V. Shirkov and I. L. Solovtsov, Phys. Rev. Lett. 79, 1209 (1997); K. A. Milton and I. L. Solovtsov, Phys. Rev. D 55, 5295 (1997)

[11] D. V. Shirkov and I. L. Solovtsov, Theor. Math. Phys. 150, 132 (2007)

[12] K. A. Milton and O. P. Solovtsova, Phys. Rev. D 57, 5402 (1998)

[13] R. S. Pasechnik, D. V. Shirkov, O. V. Teryaev, O. P. Solovtsova and V. L. Khandramai, Phys. Rev. D 81, 016010 (2010)

[14] A. V. Sidorov and O. P. Solovtsova, Nonlin. Phenom. Complex Syst. 16, 397 (2013)

[15] A. V. Sidorov and O. P. Solovtsova, Phys. Part. Nucl. Lett. 14(1), 1 (2017)

[16] L. M. Barkov et al., Nucl. Phys. B 256, 365 (1985)

[17] B. Aubert et al. (BABAR Collaboration), Phys. Rev. D 71, 052001 (2005)

[18] M. R. Whalley, J. Phys. G: Nucl. Part. Phys. 29, A1 (2003)

[19] L. M. Kurdadze et al., J. Exp. Theor. Phys. Lett. 43, 643 (1986)

[20] S. I. Dolinsky et al., Phys. Rep. 202, 99 (1991)

[21] M. N. Achasov et al., Preprint BUDKER-INP-2001-34 (2001)

[22] B. Bisello et al., Preprint LAL-90-35 (1990)

[23] G. Cosme et al., Nucl. Phys. B 152, 215 (1979)

[24] B. Aubert et al., Phys. Rev. D 73, 052003 (2006)

[25] G. J. Gounaris, J. J. Sakurai, Phys. Rev. Lett. 21, 244 (1968)

[26] K. A. Olive et al. (Particle Data Group), Chin. Phys. C 38, 1025 (2014 and 2015 update)

[27] B. V. Geshkenbein, B. L. Ioffe, and K. N. Zyablyuk. Phys. Rev. D 64, 093009 (2001)

[28] B. L. Ioffe, K. N. Zyablyuk. Eur. Phys. J. C 27, 229 (2003) 